\title{
HNRNPDL-related muscular dystrophy: expanding the clinical, morphological and MRI phenotypes
}

\section{Berardo, Andrés}

2019-10

Berardo , A , Lornage , X, Johari , M , Evangelista , T, Cejas , C , Barroso , F , Dubrovsky , A, Bui , M T , Brochier , G, Saccoliti , M , Bohm , J , Udd , B , Laporte , J, Romero , N B \& Taratuto , A L 2019 , ' HNRNPDL-related muscular dystrophy: expanding the clinical, morphological and MRI phenotypes ' , Journal of Neurology , vol. 266 , no. 10 , pp. 2524-2534 . https://doi.org/10.1007/s00415-019-09437-3

http://hdl.handle.net/10138/306750

https://doi.org/10.1007/s00415-019-09437-3

cc_by

publishedVersion

Downloaded from Helda, University of Helsinki institutional repository.

This is an electronic reprint of the original article.

This reprint may differ from the original in pagination and typographic detail.

Please cite the original version. 


\title{
HNRNPDL-related muscular dystrophy: expanding the clinical, morphological and MRI phenotypes
}

\author{
Andrés Berardo $^{1} \cdot$ Xavière Lornage $^{2} \cdot$ Mridul Johari $^{3,4} \cdot$ Teresinha Evangelista $^{5,6} \cdot$ Claudia Cejas $^{7} \cdot$ Fabio Barroso $^{7}$. \\ Alberto Dubrovsky ${ }^{8}$. Mai Thao Bui ${ }^{5} \cdot$ Guy Brochier $^{5,6} \cdot$ Maria Saccoliti $^{9} \cdot$ Johann Bohm $^{2} \cdot$ Bjarne Udd $^{4,10}$. \\ Jocelyn Laporte ${ }^{2} \cdot$ Norma Beatriz Romero $^{5,6} \cdot$ Ana Lia Taratuto $^{9}$
}

Received: 23 March 2019 / Revised: 16 June 2019 / Accepted: 18 June 2019 / Published online: 2 July 2019

c) Springer-Verlag GmbH Germany, part of Springer Nature 2019

\begin{abstract}
Autosomal dominant limb girdle muscular dystrophy D3 HNRNPDL-related is a rare dominant myopathy caused by mutations in HNRNPDL. Only three unrelated families have been described worldwide, a Brazilian and a Chinese carrying the mutation c.1132G $>$ A p.(Asp378Asn), and one Uruguayan with the mutation c.1132G $>$ C p. (Asp378His), both mutations occurring in the same codon. The present study enlarges the clinical, morphological and muscle MRI spectrum of AD$H N R N P D L$-related myopathies demonstrating the significant particularities of the disease. We describe two new unrelated Argentinean families, carrying the previously reported c.1132G $>\mathrm{C}$ p.(Asp378His) HNRNPDL mutation. There was a wide phenotypic spectrum including oligo-symptomatic cases, pure limb girdle muscle involvement or distal lower limb muscle weakness. Scapular winging was the most common finding, observed in all patients. Muscle MRIs of the thigh, at different stages of the disease, showed particular involvement of adductor magnus and vastus besides a constant preservation of the rectus femoris and the adductor longus muscles, defining a novel MRI pattern. Muscle biopsy findings were characterized by the presence of numerous rimmed vacuoles, cytoplasmic bodies, and abundant autophagic material at the histochemistry and ultrastructural levels. $H N R N P D L$-related LGMD D3 results in a wide range of clinical phenotypes from the classic proximal form of LGMD to a more distal phenotype. Thigh MRI suggests a specific pattern. Codon 378 of $H N R N P D L$ gene can be considered a mutation hotspot for HNRNPDL-related myopathy. Pathologically, the disease can be classified among the autophagic rimmed vacuolar myopathies as with the other multisystem proteinopathies.
\end{abstract}

Keywords LGMDD3 HNRNPDL-related $\cdot H N R N P D L$ gene $\cdot$ Rimmed vacuolar myopathy $\cdot$ Autophagy

Norma Beatriz Romero

nb.romero@institut-myologie.org

1 Neuropsychiatry Center Valencia Positiva, Córdoba, Argentina

2 Department of Translational Medicine, Institut de Génétique Et de Biologie Moléculaire Et Cellulaire (IGBMC)INSERM U1258, UMR7104, Strasbourg University, Illkirch, France

3 Department of Medical Genetics, University of Helsinki, Medicum, Finland

4 Folkhälsan Research Center, Helsinki, Finland

5 Morphology Unit, Myology Institute, GHU Pitié-Salpêtrière, 75013 NeuromuscularParis, France
6 Sorbonne Université, AP-HP, INSERM, Centre de référence Des Maladies Neuromusculaires Nord/Est, Ile de France, Paris, France

7 Instituto de Investigaciones Neurológicas FLENI, Buenos Aires, Argentina

8 Fundación Favaloro, Buenos Aires, Argentina

9 Neuropathology and Neuromuscular Diseases Laboratory, Buenos Aires, Argentina

10 Neuromuscular Research Center, Tampere University and University Hospital, Tampere, Finland 


\section{Introduction}

According to the recent definition established by a group of experts at the 229th European Neuromuscular Centre (ENMC)international workshop in 2017: 'Limb girdle muscular dystrophy (LGMD) is a genetically inherited condition that primarily affects skeletal muscle leading to progressive, predominantly proximal muscle weakness at presentation' [1]. Among LGMD, the autosomal dominant forms are relatively rare and represent $10 \%$ of all LGMD [2]. According to the new LGMD classification, autosomal dominant LGMD includes: LGMDD1 DNAJB6-related, LGMDD2 TNPO3-related, LGMDD3 HNRNPDL-related and LGMDD4 CAPN3-related myopathy [1].

LGMDD3 HNRNPDL-related, previously LGMD1G (OMIM \#609115) was first described in 2004 in a dominant Brazilian-Caucasian family with 12 patients distributed over three generations [2]. The age at onset varied from 30 to 47 years. The slowly progressive disease started with proximal lower limbs involvement later followed by proximal upper limbs weakness. Progressive finger and toe flexion limitations and muscle cramps were present in most patients. Serum creatinekinase (CK) activity was normal or slightly increased. Muscle histology was available for one patient and described as a "myopathic pattern" with fibre size variability, necrotic fibres, groups of atrophic angulated fibres, and rimmed vacuoles [2]. In 2014, LGMDD3HNRNPDL-related was also reported in a Uruguayan family with 18 affected members [3].The age of onset varied from 15 to 55 years of age. The clinical signs were similar to those of the Brazilian family, but in 8 patients the symptoms started with proximal upper limb weakness and difficulty to raise the arms. Additionally, some affected members presented early onset cataracts [3].For both the Brazilian and Uruguayan families; the disease affected both sexes with incomplete penetrance. Genome sequencing identified two different missense mutations in HNRNPDL (on chromosome 4q21) encoding the heterogeneous nuclear ribonucleoprotein D-like (hnRNPDL). Both mutations were heterozygous and affected the same aminoacid: p.(Asp378Asn) in the Brazilian family and p.(Asp378His) in the Uruguayan family. Recently, a Chinese family with 10 affected individuals over three generations was reported to carry the original Brazilian p.(Asp378Asn) mutation [4].While muscle morphology showed similar features with the presence of rimmed vacuoles, the clinical phenotype slightly differed from the previously reported families with half of the Chinese patient's presenting distal as well as proximal limb weakness. Flexion limitation of fingers and toes, which was a typical feature in the other two reported families, was not present, highlighting the broad clinical spectrum of the disease.

hnRNPDL acts as a regulator of pre-mRNA splicing and nuclear export. HNRNPDL mutations are thought to cause defects in mRNA biogenesis and metabolism [5].This DNA/RNA-binding protein has been shown to localize in the cytoplasm and in the nucleus [6, 7]. Data from Vieira et al. 2014, suggested that the translocation of the protein from the cytoplasm to the nucleus might be impaired by the p.(Asp378Asn)mutation. This could prevent the protein to access to the nucleus to perform its function on premRNA maturation. Many hnRNPs were linked to various diseases due to their crucial role in the regulation of gene expression [8]. Mutations in the prion-like domains (PrLD) of hnRNPA1 and hnRNPA2B1 cause a multisystem proteinopathy (MSP), an inherited pleiotropic degenerative disorder that can affect muscle, bone, and the nervous system. However, an isolated myopathy has also been described in association with hnRNPA1 mutations [9]. Affected tissues in multisystem proteinopathies present ubiquitin-positive inclusions that contain RNA-binding proteins, such as hnRNPA1 and hnRNPA2B1, and may stain positive for proteins that mediate ubiquitin-dependent autophagy [9]. Rimmed vacuolation is a key feature of the myopathology in MSP [10].

Here we describe two unrelated families from Argentina with LGMDD3 HNRNPDL-related at the clinical, muscle imaging, histopathological and ultrastructural level.

\section{Materials and methods}

\section{Patients}

Two unrelated Argentinean families of European ancestry with history of adult-onset myopathy were included in the study (Fig. 1: family A and family B). All patients underwent detailed clinical examination summarized in Table1.

\section{Morphological studies}

Open muscle biopsies of the quadriceps were obtained from four patients, three (III-1, II-6 and II-9) from family A (FA) and one (III-1) from family B (FB) (Fig. 1). For conventional histochemical techniques $10-\mu \mathrm{m}$ thick cryostat sections were stained with haematoxylin and eosin (HE), modified Gomori trichrome (mGT), Periodic-acid Schiff technique (PAS), Oil red $\mathrm{O}$, nonspecific esterase, reduced nicotinamide adenine dinucleotide dehydrogenase-tetrazolium-reductase(NADHTR), succinic dehydrogenase (SDH), cytochrome c oxidase (COX), menadione-nitro blue tetrazolium and adenosine triphosphatase (ATPase) pre-incubated at $\mathrm{pH} 9.4,4.63,4.35$. 


\section{Family A}

।

II

III<smiles>CCCC1CC1C</smiles><smiles>CC1CCC(C)C(C2CC3CC2C3)C1</smiles><smiles>C1CCCC1</smiles>

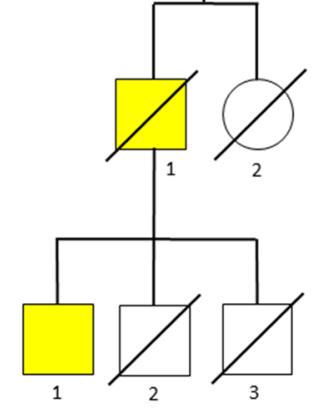

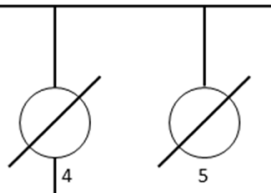
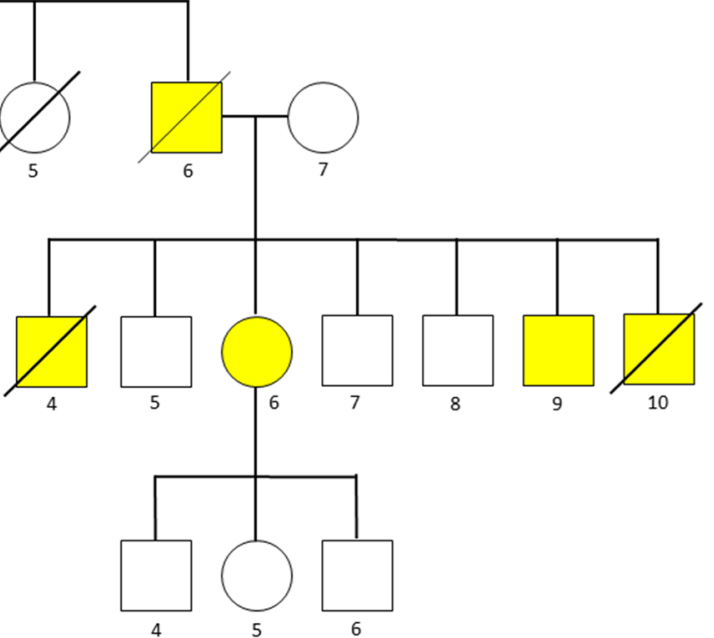

\section{Family B}

I

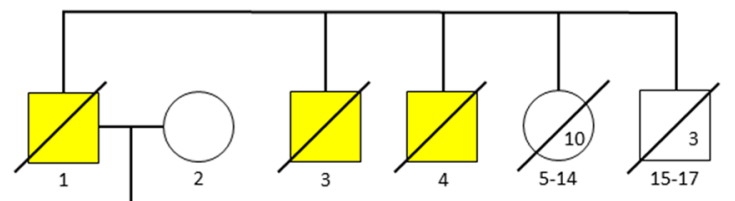

II

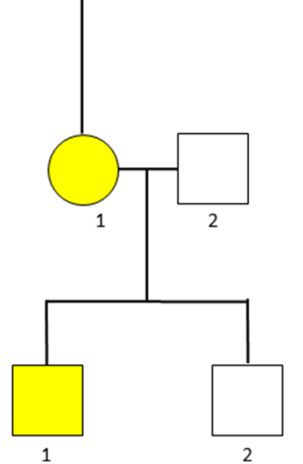

Fig. 1 Family A and Family B pedigrees

\section{Immunohistochemistry (IHC)}

IHC was performed in frozen muscle sections from patients III-1, II-6, II-9from FA. Antibodies against Desmin (AntiHuman Desmin, Clone D33, Dako Laboratories, Denmark A/S), Myotilin (NCL-Myotilin, Novocastra Laboratories, Newcastle Upon Tyne, United Kingdom), $\alpha$ B-crystallin (CRYAB, GeneTex International Corporation, Irvine, USA), C5b9 (Dako), P62 (BD-Transduction Laboratories) and antibodies against dystrophin (Dys2, Leica), a, b, g, d sarcoglycans (Leica), alpha 2 laminin, dysferlin (Leica), were applied to $10 \mu \mathrm{m}$ thick cryosections and revealed using immunoperoxidase techniques.

\section{Electron microscopy}

Electron microscopy (EM) was performed in three cases (FA.III-1,FA.II-6 and FB.III-1).Small muscle samples were fixed in $2.5 \%$ glutaraldehyde, $\mathrm{pH} 7.4$, post-fixed in $4 \%$ osmium tetroxide, dehydrated and embedded in araldite. Several blocks from each patient were studied, including longitudinally and transversally oriented samples. Semi-thin sections were stained with toluidine-blue and examined on light microscopy. Ultrathin sections were stained with uranyl acetate and lead citrate (Reynolds) and viewed with a Zeiss EM 109 T. 


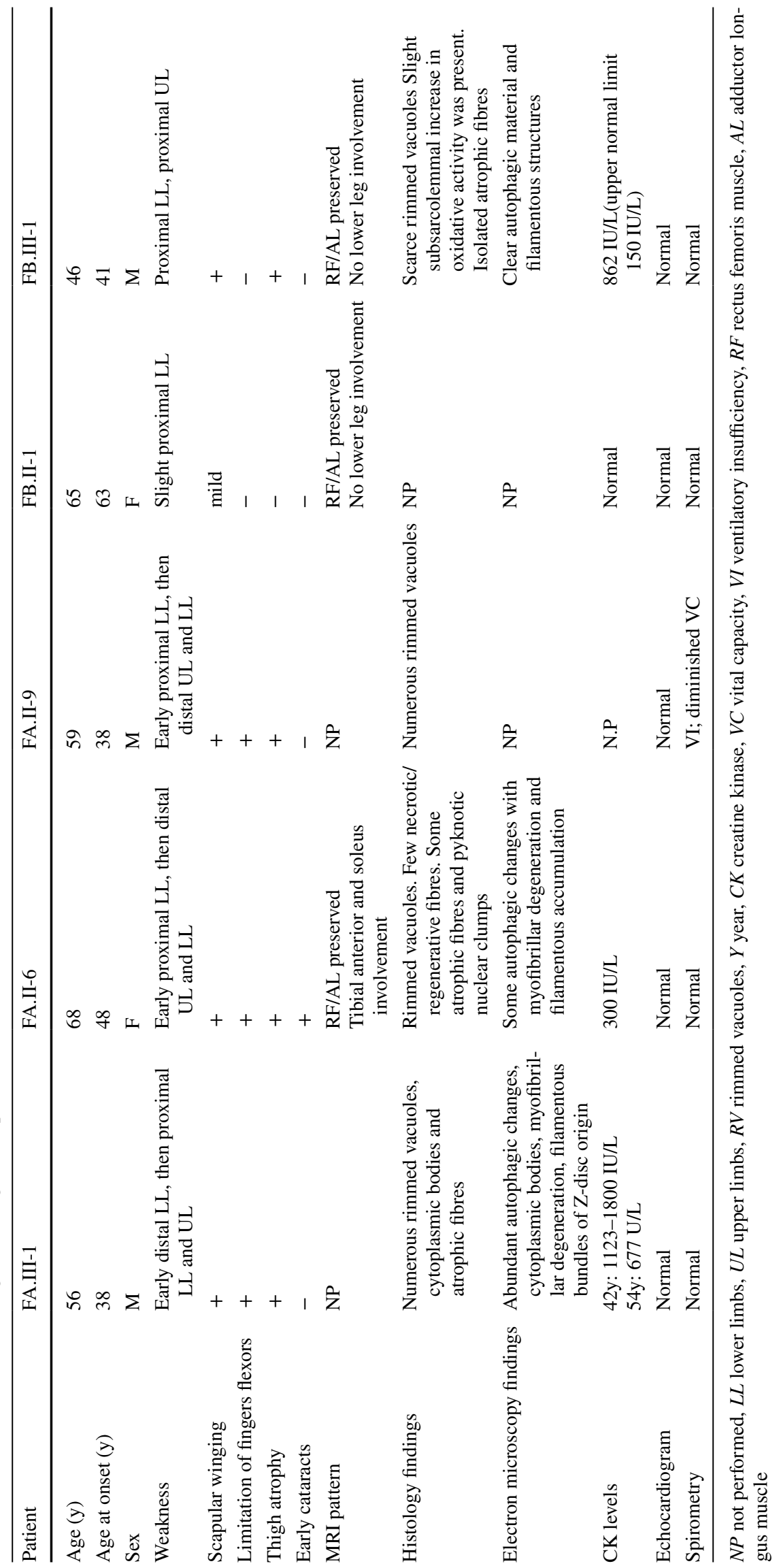




\section{Genetic analysis}

In family A (Fig. 1), exome sequencing was performed for individuals FA.II-6, FA.II-7 and FA.II-9 with the SureSelect Human All Exon $50 \mathrm{Mb}$ capture library v5 (Agilent, Santa Clara, USA). Samples were paired-end sequenced on Illumina HiSeq 2500 (Illumina, San Diego, USA). In family $\mathrm{B}$ (Fig. 1), exome sequencing was performed on the index patient (FB.III-1) with Roche SeqCap EZ Human Exome v3 with 75 bp paired end reads and sequenced on Illumina NextSeq 550 (Illumina, San Diego, USA).

Alignment was performed on the GRCh37/hg 19 reference genome and the variants were filtered using their frequency in gnomAD (https://gnomad.broadinstitute.org/) and in our in-house database containing over 1500 exomes. The variants with minor allele frequency over 0.01 were removed from analysis. The mutation was numbered according to the GenBank NM_031372.3 with +1 corresponding to the A of the ATG translation initiation codon.

In family A, confirmation of variants and segregation was performed with Sanger sequencing for four affected (FA. II-6, FA.II-9, FA.II-10, FA.III-1) and three healthy individuals (FA.II-5, FA.II-7, FA.III-2). In family B, the segregation was verified by Sanger sequencing in three other family members (FB.II-1, FB.II-2 and FB.III-2).

\section{Magnetic resonance imaging (MRI)}

Muscle MRI was performed in one member from family A (FA.II-6) and two from family B (FB.III-1, FB.II-1). Fast Spin Echo (FSE) T1 and T2-weighted STIR (Short-tau inversion recovery) images were acquired in axial and coronal planes of both thighs and legs. We analysed and classified all cases considering the type of involvement of each muscle: perifascial, concentric, mottled, muscle atrophy vs. hypertrophy, oedema vs. fatty infiltration, the muscles most affected in each compartment, using the Mercuri score [11, 12].

\section{Results}

\section{Phenotypic presentation and clinical investigations}

\section{Family A (FA)}

An Argentinean family originally from the province of Buenos Aires with Italian ancestry. There were 10 affected family members over 3 generations (Fig. 1). All affected individuals had a normal early motor development. The age at onset ranged from 38 to 48 years. The initial symptoms were proximal lower limb weakness in two patients (FA.II-6, II-9) associated with distal lower limb weakness in one of them (FA.III-1). Scapular winging and anterior thigh atrophy were common findings in all patients (Fig. 2). Other symptoms and signs were muscle cramps (FA. I-6, II-6 and II-10) and early onset cataracts (FA. I6, II-4, II-6 and II-10). No additional clinical information was available from the deceased relatives.

\section{FA.III-1}

A 56-year-old male with asymmetric distal lower limb weakness from the age of 38 , unable to walk on heels. Muscle weakness progressed to the proximal lower limb muscles, and to the shoulder and arm muscles. This resulted in frequent falls. Later on, he developed limitations of finger and toe flexors. At present he is unable to raise his arms, has marked bilateral scapular winging (Fig. 2b), symmetrical quadriceps atrophy and walks with a cane. No cataracts were observed. CK levels were variably increased at several determinations, with values between 677-1800 IU/1 [normal values (nv): 55-170 IU/l]. Cardiovascular and pulmonary evaluation was unremarkable. He had two non-affected brothers that are deceased. His deceased father developed scapular winging during adulthood and cataracts at 70 years of age.

\section{FA.II-6}

A 68-year-old woman whose first symptom was early onset cataracts revealed at the age of 42 years. By the age of 48 , she developed proximal lower limb weakness, progressive difficulty in climbing stairs and frequent falls. Later on, the weakness progressed to distal muscles with movement limitation affecting first the toes and later the finger flexors and small hand muscles. Scapular winging was also present (Fig. 2e).Currently she has both proximal and distal lower limb weakness (MRC 3/5), walks with a cane, and experiences frequent cramps in her feet, legs, and hands. She had a medical history of hypothyroidism controlled with medication. The CK levels were slightly elevated at $300 \mathrm{IU} / \mathrm{l}$ (nv: 30-135 IU/l). The EMG showed a "myopathic" pattern consistent with a distal involvement with normal nerve conduction studies (NCS).

\section{FA. II-9}

A 59-year-old man, started at the age of 38 with proximal and later on distal muscle weakness. Similar to the other patients he presented with limitation of toes and later finger flexors; and scapular winging (Fig. 2a). He has a normal cardiovascular evaluation; reduced forced vital capacity with ventilatory insufficiency. 


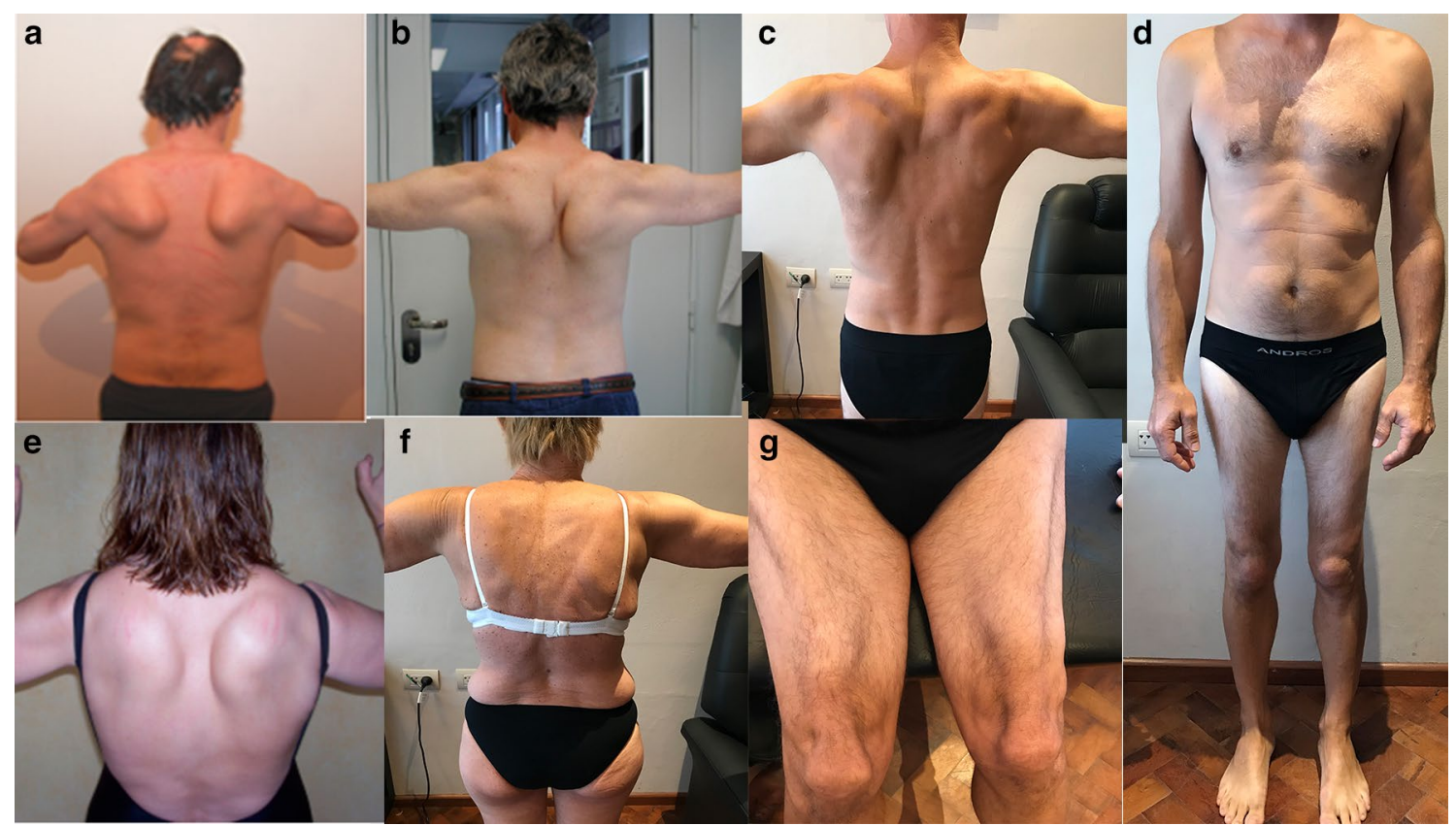

Fig. 2 Scapular winging and vastus atrophy are common clinical findings. Scapular winging was a common finding in patients: FA.II-9 (a), FA.III-1 (b), FB.III-1 (c), FA.II-6 (e) andFB.II-1 (f). Severe thigh atrophy was present in all patients (patient B.III-1) (d, g). In case

\section{Family B (FB)}

An Argentinean family from the province of Entre Rios, with French-Basque ancestry with 5 affected members over three generations (Fig. 1). Age at onset varied from 41 to 63-yearold with progressive proximal lower limb muscle weakness. There was no history of early onset cataracts or restriction of finger and toe flexion with reduced range of movement in the interphalangeal joints.

\section{FB.III-1}

A 46-year-old man, semi-professional cyclist, started to experience progressive weakness and wasting of the quadriceps from the age of 41 . He has difficulty standing from the crouched position, has occasional unexplained falls and some difficulty climbing stairs. Cardiopulmonary examination was normal. The maternal grandfather and three great uncles were affected by a similar, late onset, muscle condition. At the time of his first examination at age 41 , he had no shoulder girdle or upper arm muscles weakness but a mild bilateral scapular winging; prominent atrophy of the vastus lateralis and medialis and a striking preservation of the rectus femoris (knee extension: MRC power grade 4-/5) (Fig. 2d,g). Atrophy of the posterior thigh compartment was noticed as well as abdominal weakness. Pelvic girdle weakness was present with hip adduction and abduction being
B.II-1 (f) although she carries the mutation, slight symptoms were present. Marked atrophy of vastus muscles were observed with preservation of the rectus femoris (Patient B.III-1) (g)

compromised (MRC power grade 4+/5). Muscle strength was preserved in the lower legs and there was calf hypertrophy. Five years after the first evaluation a more pronounced scapular winging (Fig. 2c) and shoulder weakness with no lower limb distal weakness was observed. No finger or toes flexion limitation was present. The CK levels were mildly elevated (862 U/l; nv: 55-170 UI/1) and the electromyography was consistent with a "myopathic" pattern.

\section{FB.II-1 case}

The mother of the proband, a 65-year-old woman, showed a slight weakness of the vastus muscles (Knee extension MRC 4+/5), mild and asymmetrical scapula displacement (Fig. 2f) and lumbar scoliosis. The patient reports occasional lower limb cramps and for the last 3 years some difficulties climbing stairs. The CK level was normal. There is no history of early onset cataracts.

\section{Muscle biopsies}

\section{Histochemistry}

Quadriceps muscle biopsies from patients FA.III-1, FA.II6, FA.II-9, FB.III-1 at the age of 42, 55, 40 and 41 yearsold, respectively, showed the presence of rimmed vacuoles, autophagic vacuoles and cytoplasmic bodies (Fig. 3). There 

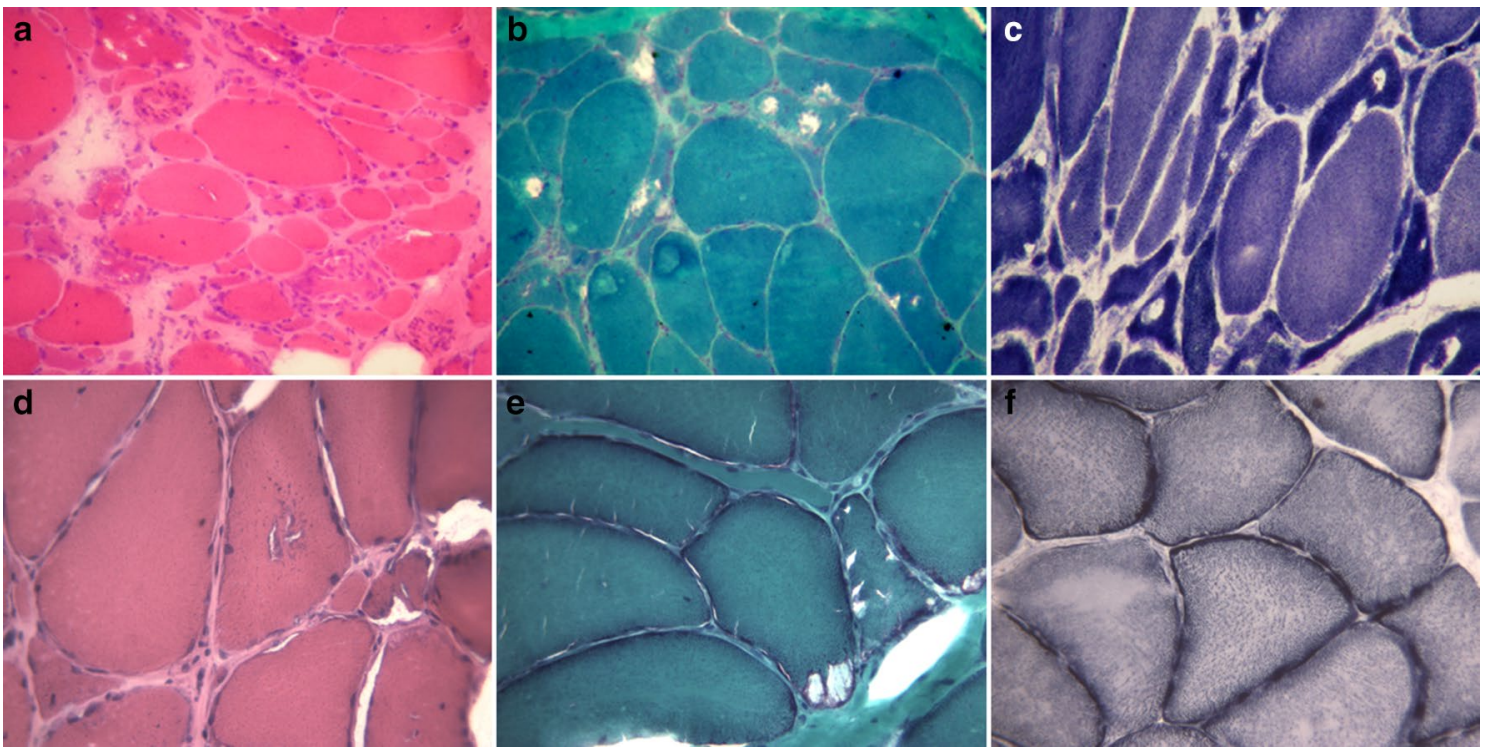

Fig. 3 Histochemistry. Frozen muscle sections from patients: FA. III-1 (a-c) and FB. III-1 (d-f). Marked variability of fibre size with some atrophic fibres. Numerous rimmed vacuoles and abundant

was variability in muscle fibre size with the presence of atrophic fibres and a few pyknotic nuclear clumps. There was a moderate increase in the endomysial/perimysial connective tissue and in one case, some necrotic/regenerating fibres were observed.

\section{Immunohistochemistry (IHC)}

Dystrophin; lamininalpha-2; alpha, beta, gamma, and delta sarcoglycans labelling was preserved. ForFA.II-9: Some of the vacuoles were labelled with antibodies for dystrophin, thus confirming the presence of sarcolemma membranes in the vacuoles as previously described. IHC study for desmin, myotilin, and $\alpha \mathrm{B}$-crystallin did not show any specificity, with only an inconsistent and unspecific immunostaining of a few degenerating fibres. Some vacuoles were labelled with the antibodies against $\mathrm{C} 5 \mathrm{~b} 9$ and dystrophin and a good number of muscle fibres presented accumulations of P62.

\section{Ultrastructural study}

Electron microscopy studies were performed in three muscle biopsies (FA.III-1,FA.II-6 and FB.III-1) (Fig. 4). All biopsies showed marked autophagic changes, particularly in patients FA.III-1 and FB.III-1 and less prominent in patient FA.II-6. We could observe numerous vacuoles containing membranous remnants, myeloid bodies, and cytoplasmic bodies (Fig. 4b, c, f). The myofibrils were regularly dissociated by amorphous material from the autophagy debris. Cytoplasmic areas with tubulofilamentous inclusions, a autophagy lesions were observed in many fibres: a (HE), b (GT), d (HE) and e (GT). Vacuoles and some areas of disorganized structure were also detected with oxidative techniques: $\mathrm{c}$ and $\mathrm{f}$ (NADH-TR)

structural marker of rimmed vacuoles, were detected in some muscle fibres (Fig. 4a, d). Additionally, remaining segments of disrupted sarcomeres were present in the disorganized regions of the fibres, and small areas of dispersed filamentous bundles of Z-disc origin were also noted (Fig. 4e). Of note that, neither granular filamentous aggregates nor nuclear tubulofilamentous inclusions were observed in these muscle biopsies.

\section{Genetic analysis}

By exome sequencing, we identified the same heterozygous HNRNPDL mutation in both families. This missense mutation, c. $1132 \mathrm{G}>\mathrm{C}$ p.(Asp378His), affects a conserved amino acid encoded by exon 6 of HNRNPDL. The mutation was not listed in gnomADor in our in-house database containing over 1500 exomes and was previously reported as causing LGMDD3. In family A, the presence of the HNRNPDL mutation in the patients and its absence in the healthy family members was confirmed by Sanger sequencing. In F.B, HNRNPDL: c. 1132G >C p.(Asp378His) was found in the index patient as well as the mother, whereas the father and the healthy brother did not harbour the mutated allele.

\section{MRI findings}

MRI findings showed in the three cases analysed at the thighs, a selective pattern involving heavily adductor magnus and vastus muscles with preservation of rectus femoris and adductor longus (Fig. 5). Lower distal leg involvement 


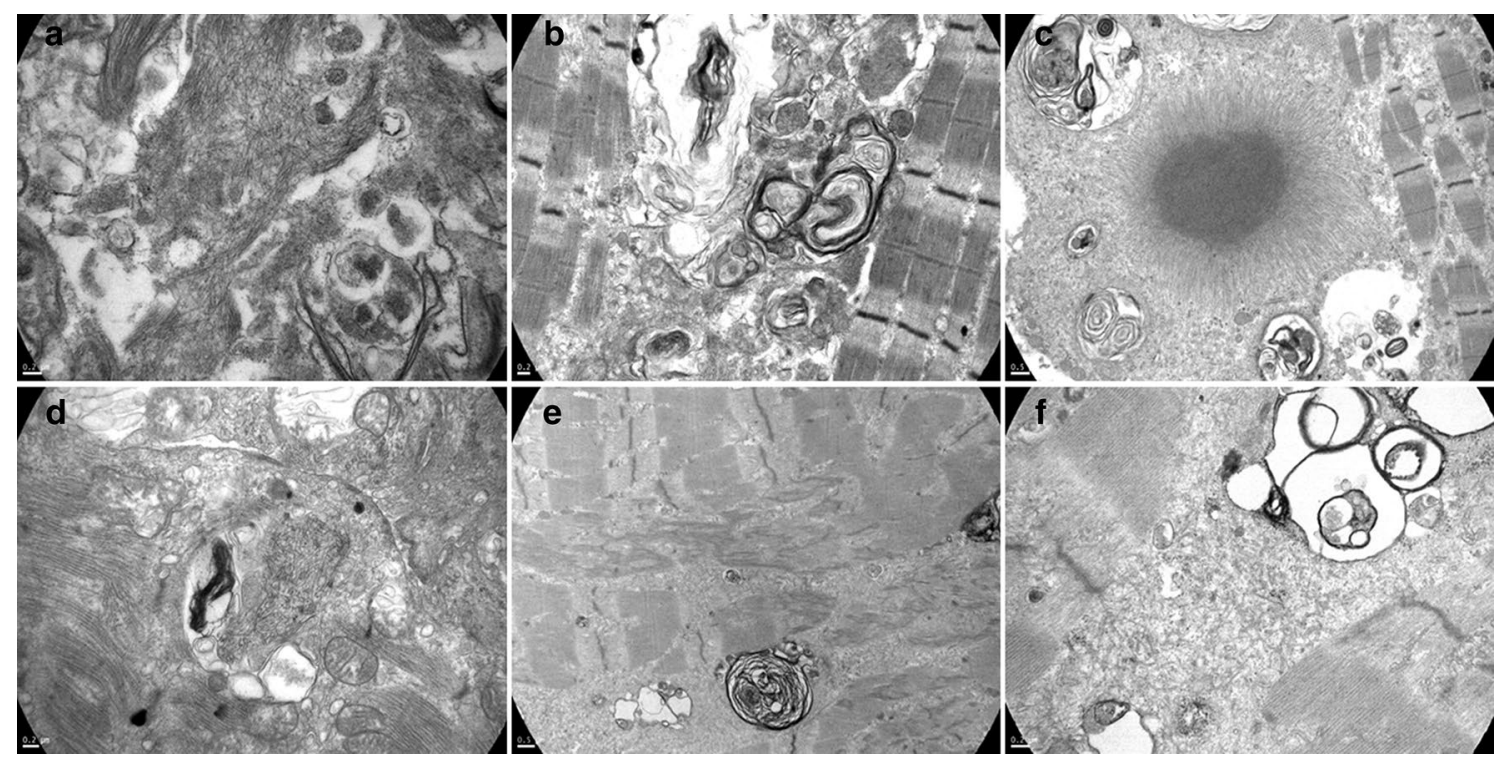

Fig. 4 Ultrastructural findings. Longitudinal muscle sections from patients: FA. III-1 (a-c) and FB. III-1 (d-f). The autophagic vacuoles containing membranous remnants, myeloid bodies and some glycogen granules $(\mathbf{b}, \mathbf{f})$ are observed among the myofibrils. Cytoplasmic

was present only in one patient showing replacement of anterior compartment and soleus muscles.

\section{FA. II-6}

Muscle MRI shows a diffuse, symmetric, fatty infiltration of the gluteus minimus and ilio-psoas muscles; moderate fatty infiltration of the gluteus maximus predominantly on the right and of the tensor fascia lata with relative sparing of gluteus medium muscles. At the thighs, on the anterior compartment, a selective involvement of vastus medialis, intermedius and lateralis, sartorius, adductor magnus, with relative sparing of the rectus femoris and adductor longus muscles were observed (Fig. 5e). On the posterior compartment there was a severe involvement of the semitendinosus with relative sparing of the semimembranosus and long head of biceps femoris. At the lower legs the tibial anterior, extensor hallucis and soleus were affected (Fig. 5f).

\section{FB.III-1}

Thigh MRI showed severe fatty infiltration of all vastus muscles, sartorius and adductor magnus with relative sparing of gracilis, biceps femoris, semitendinosus, and semimembranosus muscles. Preservation of rectus femoris and adductor longus were observed with hypertrophy of rectus femoris (Fig. 5c). At the lower leg there was no evident muscle involvement (Fig. 5d). bodies (c) and few zones with spread filamentous bundles of Z-disc material (e) were also noted. Areas with tubulofilamentous inclusions, a structural marker of rimmed vacuoles, were detected in some muscle fibres (a, d)

\section{FB. II-1}

Severe fatty infiltration was observed in the vastus lateralis, adductor magnus and sartorius muscles with relative sparing of rectus femoris and the adductor longus (Fig. 5a). Semitendinosus, semimembranosus and biceps femoris were moderately affected. At the lower leg level, no evident involvement was detected (Fig. 5b).

\section{Comparative MRI studies}

Comparative MRI studies from both FA. II-6 and FB.III-1 patients were performed with an interval of 12 years and 4 years, respectively (Fig. 6).

\section{Discussion}

LGMDD3 HNRNPL-related is caused by heterozygous mutations in the HNRNPDL gene. Until now, only three families were described worldwide: a Brazilian-Caucasian, a Uruguayan, and a Chinese family [2-4]. The missense mutations in HNRNPDL affected the same aminoacid in all families although the transition is different: p.(Asp378Asn) in the Brazilian and Chinese families and p.(Asp378His) in the Uruguayan family. The description of several families carrying the mutation in the same amino acid could define a mutational hotspot.

This study describes two unrelated Argentinian families with European ancestry (Italian/French-Basque) with 


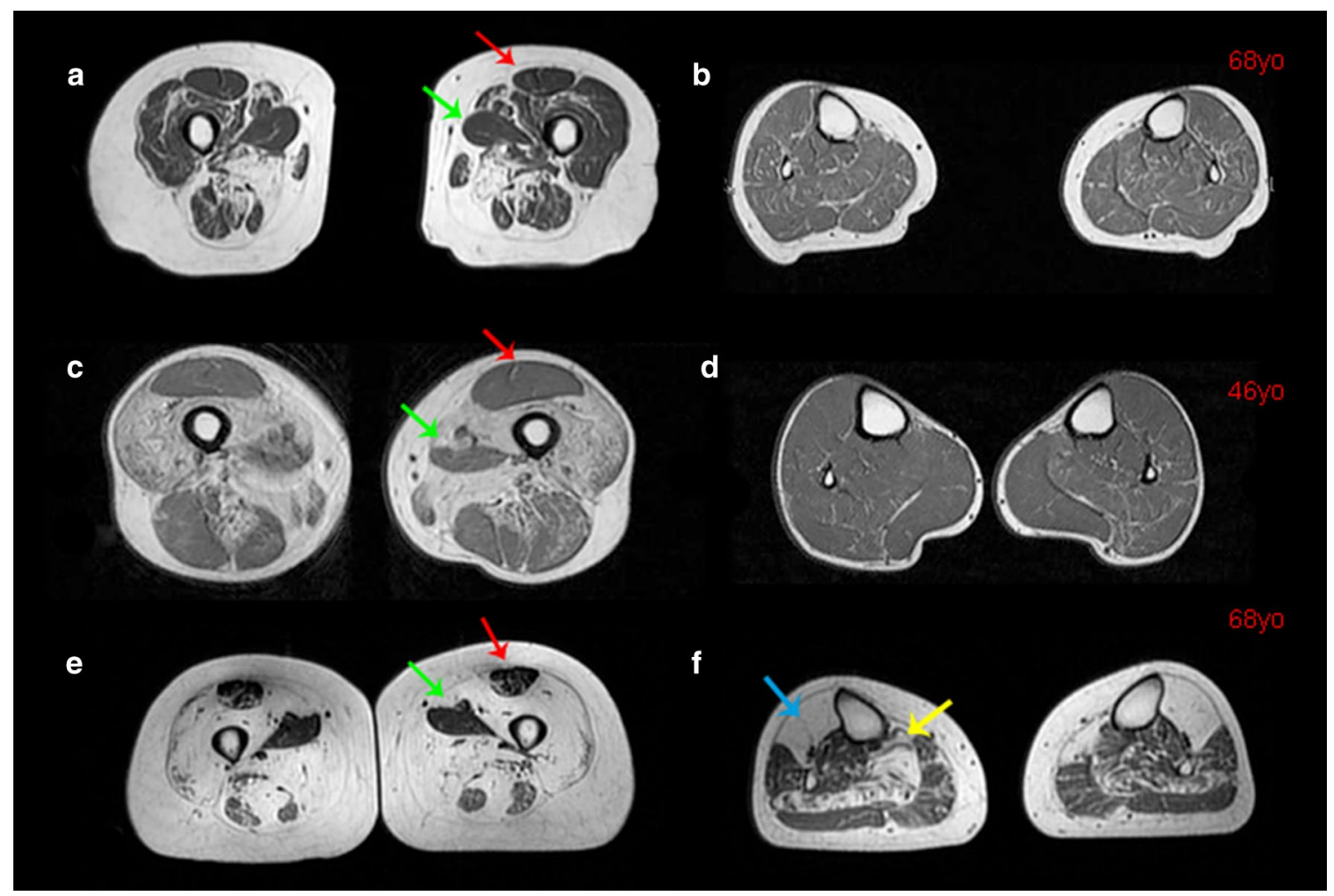

Fig. 5 MRI reveals a specific muscle pattern at the thigh. T1 weighted MRI sections shows preservation of adductor longus and rectus femoris as a common finding in all patients, even in different stages of the disease. a Thigh MRI Patient FB.II-1 performed at age 68; severe fatty infiltration was observed on the vastus lateralis, adductor magnus and sartorius muscles with relative sparing of rectus femoris (arrow) and the adductor longus (arrow). Semitendinosus, semimembranosus and biceps femoris were moderately affected. b At the leg level no evident involvement was observed. c Thigh MRI in patient FB.III-1 performed at age 46 showed severe fatty infiltration of vasti muscles, Sartorius and adductor magnus with relative sparing of gracilis, biceps femoris, semitendinosus and semimembrano- sus muscles. Preservation of rectus femoris and adductor longus were observed with hypertrophy of rectus femoris. d At the leg level no evident involvement was observed. e Muscle MRI in patient FA.II-6 performed at 68-year-old. At the thigh level, almost complete fatty replacement is observed with selective involvement of vastus medialis, intermedius and lateralis, sartorius and adductor magnus with relative sparing of the rectus femoris and adductor longus. In the posterior compartment, severe involvement of semitendinosus was seen, with relative sparing of semimembranosus and long head of biceps femoris. f At the legs, tibial anterior (arrow), extensor hallucis and soleus (arrow) were also affected

descriptions regarding variable clinical severity of the disease. In our cohort, as in previous reported series, the myopathy usually manifested as a late onset slowly progressive disorder, the age of onset ranging from 38 to 63 years. Normal cardiac and respiratory assessments in most of the studied patients could suggest that cardiac and respiratory insufficiency may not be a feature of this condition, however, the oldest patient in this series presents a reduction of the FVC over time with a pattern that may suggest the diaphragm is compromised. We, therefore, suggest that the respiratory function should be monitored in this condition. Some clinical similarities can be established between LGMD D3 HNRNPDL-related and myopathies associated with mutations in other genes coding for other heterogeneous nuclear ribonucleoproteins (hnRNPs) such as hnRNPA1. Rimmed vacuolar myopathy has been documented with mutations in hnRNPA1, causing a similar autosomal dominant, late onset 


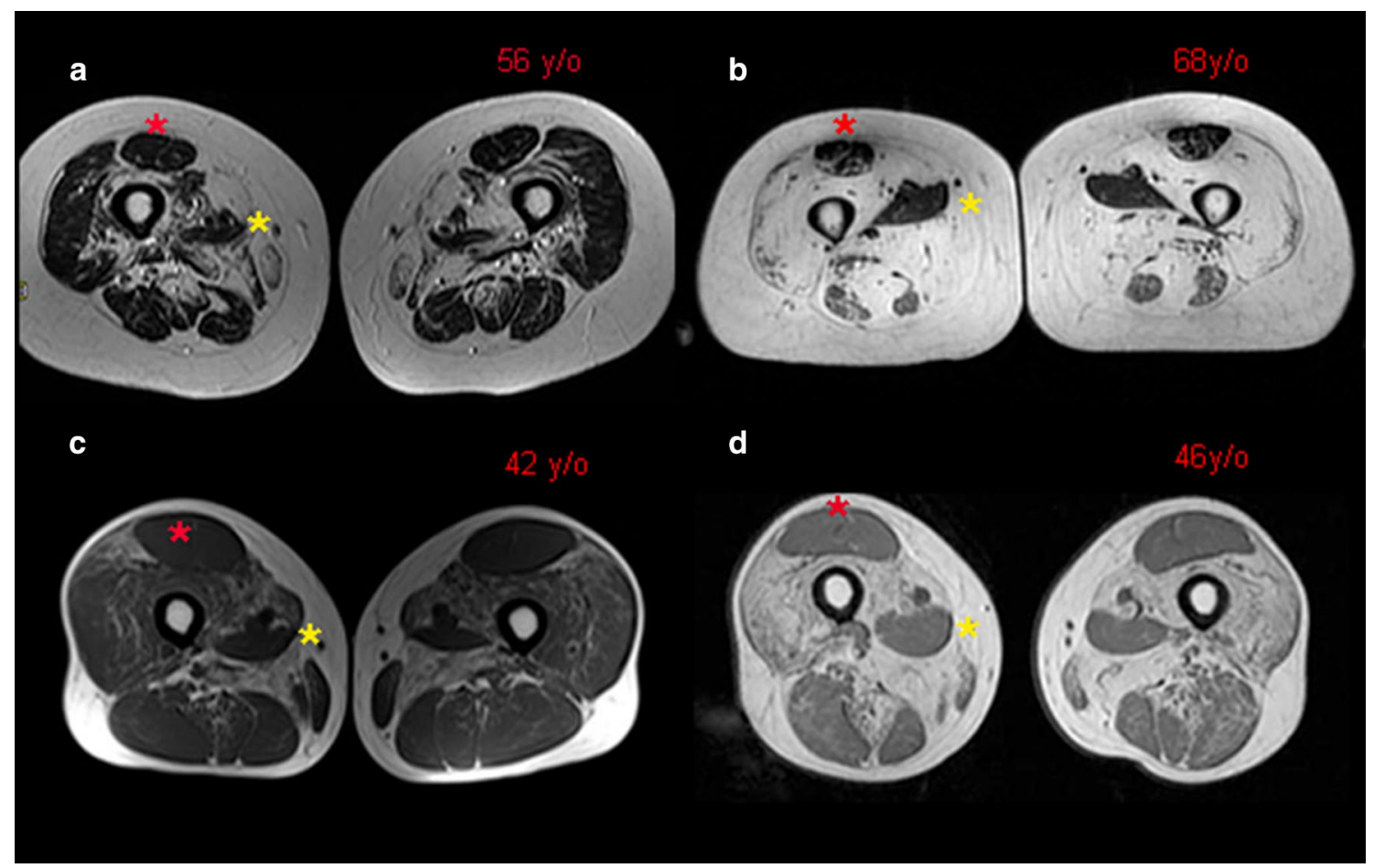

Fig. 6 Comparative thigh MRI in two different patients. T1 weighted comparative thigh MRI performed in patient FA.II-6 (56-year-old and 68-year-old) (a, b) and FB.III-1 (42-year-old and 46-year-old) (c, d) which illustrates selective sparing of rectus femoris (asterisk) and adductor longus (asterisk) through the years with marked degeneration of vasti. Posterior compartment muscles in case FA.II-6 were severely affected with the exception of semimembranosus and biceps femoris whereas in FB.III-2 are less affected myopathy with distal or proximal lower limb onset and no cardiac or respiratory involvement $[9,10,13]$.

Our MRI findings allow us to postulate a specific and pathognomonic pattern of involvement of the thighs with preservation of the rectus femoris and adductor longus, even at late stages of the disease (Figs. 5, 6).These findings are in accordance with the previous description by Sun et al. (2019), showing a strikingly similar muscle MRI pattern. Vastus muscles seem to be early and particularly affected and probably may correlate with the early falls of the patients. In contrast, no leg-specific muscle involvement was observed. A similar muscle MRI pattern, with preservation of the rectus femoris was described previously in MSPs like hnRNPA1 and hnRNPA2B1 associated diseases [9]. The reason for the selective involvement of some particular muscles with relatively sparing of others remains unclear. We could speculate that a possible association of myotoxicity with the levels of mutant HRNPNDL in different muscles might be related with the selective muscle involvement, or some muscles being more dependent on correct mRNAprocessing than others. Selective muscle involvement is not a specific finding of LGMD D3 HNRNPDL-related, but rather a common finding in muscular dystrophies. Previous reports demonstrated significant variations in isoform and gene expression levels in anatomically different muscles as one of the possible explanations of this discrepancy [14]. Other factors remain to be clarified.

The muscle biopsy studies allowed us to demonstrate a particular morphological pattern characterized by fibres harbouring numerous autophagic rimmed vacuoles and cytoplasmic bodies. Electron microscopy, performed for the first time in HNRNPDL-related LGMD D3, demonstrated autophagic changes with numerous vacuoles containing membranous remnants, myeloid bodies, besides cytoplasmic bodies and some filamentous material. Myofibrils in dissolution were separated by amorphous autophagic debris material (Fig. 4).

The morphological and clinical aspects of the $H N R N$ $P D L$-related myopathy, suggest that this entity should be included in the group of the "autophagic vacuolar myopathies" (AVM) instead of the LGMD. Of note, the cases previously reported of $H N R N P D L$-related muscular dystrophy in the literature did not include electron microscopy studies and the morphological description was very limited. These facts have led to the classification of this entity as an autosomal dominant muscular dystrophy mostly based on the clinical phenotype. With the new findings in electron microscopy that we report here, we postulate that the histological phenotype is compatible with an AVM and we suggest that the classification of this entity should be reconsidered. 
HNRNPDL belongs to the subfamily of the heterogeneous nuclear ribonucleoproteins (hnRNPs) essential for the maturation of newly formed heterogeneous nuclear premRNA into mRNA and the transport of mRNA. They are key proteins in the RNA metabolism and many hnRNP genes are known to cause degenerative diseases in particular with mutations in the prion-like domains (PrLD). Mutations in the PrLD of hnRNPA1 and A2B1 cause MSP [10]. Mutations in the PrLD of TIA1 cause rimmed vacuolar distal myopathies [15]. Mutations in the PrLD increase the tendency to self-aggregation.

In conclusion, HNRNPDL-related LGMD D3 results in a wide range of phenotypes from the classic proximal form of LGMD to a more distal phenotype. Codon 378 of HNRNPDL gene can be considered a mutation hotspot for HNRNPDL-related myopathy. Moreover, cataracts and limited fingers flexion may not be uniform findings. On the other hand, scapular winging is an important clinical key sign with similarity to VCP mutated MSP myopathy. The preservation of rectus femoris and adductor longus muscles is a common MRI feature at all stages of the disease. Pathologically the disease can be classified among the autophagic rimmed vacuolar myopathies as with the other MSP myopathies.

Acknowledgements This work has been financially supported by Association Françaisecontre les Myopathies (AFM-Telethon), Association Institut de Myologie (AIM), Assistance Publique-Hôpitaux de Paris (AP-HP), Institut National de la Santé et de la RechercheMédicale (INSERM), Sorbonne Université, Centre National de la RechercheScientifique (CNRS), University of Strasbourg, the France Génomique National infrastructure funded as part of the Investissementsd' Avenir program managed by the AgenceNationale pour la Recherche (ANR-10-INBS-09) and by Fondation Maladies Rares within the frame of the "Myocapture" sequencing project, the Fondation pour la RechercheMédicale.

\section{Compliance with ethical standards}

Conflicts of interest All authors have reviewed the manuscript. Authors have no conflict of interest to declare.

Ethical standards The study was conducted after receiving written informed consent from patients in accordance to the declaration of Helsinki.

\section{References}

1. Straub V, Murphy A, Udd B, LGMD workshop study group (2018) 229th ENMC international workshop: Limb girdle muscular dystrophies-nomenclature and reformed classification Naarden, the Netherlands, 17-19 March 2017. Neuromuscul Disord 28:702-710. https://doi.org/10.1016/j.nmd.2018.05.007

2. Starling A, Kok F, Passos-Bueno MR, Vainzof M, Zatz M (2004) A new form of autosomal dominant limb-girdle muscular dystrophy (LGMD1G) with progressive fingers and toes flexion limitation maps to chromosome 4p21. Eur J Hum Genet. 12:1033-1040

3. Vieira NM, Naslavsky MS, Licinio L, Kok F, Schlesinger D, Vainzof M, Sanchez N, Kitajima JP, Gal L, Cavacana N, Serafini PR, Chuartzman S, Vasquez C, Mimbacas A, Nigro V, Pavanello RC,
Schuldiner M, Kunkel LM, Zatz M (2014) A defect in the RNAprocessing protein HNRPDL causes limb-girdle muscular dystrophy 1G (LGMD1G). Hum Mol Genet 23:4103-4110. https://doi. org $/ 10.1093 / \mathrm{hmg} / \mathrm{ddu} 127$

4. Sun Y, Chen H, Lu Y, Duo J, Lei L, OuYang Y, Hao Y, Da Y, Shen X-M (2019) Limb girdle muscular dystrophy D3 HNRNPDL related in a Chinese family with distal muscle weakness caused by a mutation in the prion-like domain. J Neurol 266:498-506. https://doi. org/10.1007/s00415-018-9165-4

5. Li RZ, Hou J, Wei Y, Luo X, Ye Y, Zhang Y (2019) hnRNPDL extensively regulates transcription and alternative splicing. Gene 687:125-134. https://doi.org/10.1016/j.gene.2018.11.026

6. Kawamura H, Tomozoe Y, Akagi T, Kamei D, Ochiai M, Yamada M (2002) Identification of the nucleocytoplasmic shuttling sequence of heterogeneous nuclear ribonucleoprotein D-like protein JKTBP and its interaction with mRNA. J Biol Chem 277:2732-2739

7. Kamei D, Yamada M (2002) Interactions of heterogeneous nuclear ribonucleoprotein D-like protein JKTBP and its domains with highaffinity binding sites. Gene 298:49-57

8. Geuens T, Bouhy D, Timmerman V (2016) The hnRNP family: insights into their role in health and disease. Hum Genet 135:851867. https://doi.org/10.1007/s00439-016-1683-5

9. Izumi R, Warita $H$, Niihori $T$, Takahashi $T$, Tateyama M, Suzuki N, Nishiyama A, Shirota M, Funayama R, Nakayama K, Mitsuhashi S, Nishino I, Aoki Y, Aoki M (2015) Isolated inclusion body myopathy caused by a multisystem proteinopathy-linked hnRNPA1 mutation. Neurol Genet 1:e23. https://doi.org/10.1212/NXG.00000 00000000023

10. Kim HJ, Kim NC, Wang YD, Scarborough EA, Moore J, Diaz Z, MacLea KS, Freibaum B, Li S, Molliex A, Kanagaraj AP, Carter R, Boylan KB, Wojtas AM, Rademakers R, Pinkus JL, Greenberg SA, Trojanowski JQ, Traynor BJ, Smith BN, Topp S, Gkazi AS, Miller J, Shaw CE, Kottlors M, Kirschner J, Pestronk A, Li YR, Ford AF, Gitler AD, Benatar M, King OD, Kimonis VE, Ross ED, Weihl CC, Shorter J, Taylor JP (2013) Mutations in prion-like domains in hnRNPA2B1 and hnRNPA1 cause multisystem proteinopathy and ALS. Nature 495:467-473. https://doi.org/1038/nature11922

11. Mercuri E, Cini C, Pichiecchio A, Allsop J, Counsell S, Zolkipli Z, Messina S, Kinali M, Brown SC, Jimenez C, Brockington M, Yuva Y, Sewry CA, Muntoni F (2003) Muscle magnetic resonance imaging in patients with congenital muscular dystrophy and Ullrich phenotype. Neuromuscular Disord 13:554-558

12. Mercuri E, Pichiecchio A, Allsop J, Messina S, Pane M, Muntoni F (2007) Muscle MRI in inherited neuromuscular disorders: past, present, and future. J Magn Reson Imaging 25:433-440

13. Kottlors M, Moske-Eick O, Huebner A, Krause S, Mueller K, Kress W, Schwarzwald R, Bornemann A, Haug V, Heitzer M, Kirschner J (2010) Late-onset autosomal dominant limb girdle muscular dystrophy and Paget's disease of bone unlinked to the VCP gene locus. J Neurol Sci 291:79-85. https://doi.org/10.1016/j.jns.2009.12.008

14. Huovinen S, Penttilä S, Somervuo P, Keto J, Auvinen P, Vihola A, Huovinen S, Pelin K, Raheem O, Salenius J, Suominen T, Hackman P, Udd B (2015) Differential isoform expression and selective muscle involvement in muscular dystrophies. Am J Pathol 185:28332842. https://doi.org/10.1016/j.ajpath.2015.06.018

15. Lee Y, Jonson PH, Sarparanta J, Palmio J, Sarkar M, Vihola A, Evilä A, Suominen T, Penttilä S, Savarese M, Johari M, Minot MC, Hilton-Jones D, Maddison P, Chinnery P, Reimann J, Kornblum C, Kraya T, Zierz S, Sue C, Goebel H, Azfer A, Ralston SH, Hackman P, Bucelli RC, Taylor JP, Weihl CC, Udd B (2018) TIA1 variant drives myodegeneration in multisystem proteinopathy with SQSTM1 mutations. J Clin Invest 128:1164-1177. https://doi. org/10.1172/JCI97103 